\title{
Functional regulatory evolution outside of the minimal even-skipped stripe 2 enhancer
}

\author{
Justin Crocker $^{1 *}$, David L. Stern ${ }^{1}$
}

\begin{abstract}
Transcriptional enhancers are regions of DNA that drive gene expression at precise times, levels, and locations. While many studies have elucidated how individual enhancers can evolve, most of this work has focused on what are called "minimal" enhancers, the smallest DNA regions that drive expression that approximates an aspect of native gene expression. Here we explore how the Drosophila erecta even-skipped (eve) locus has evolved by testing its activity in the divergent $D$. melanogaster genome. We found, as has been reported previously, that the minimal $D$. erecta eve stripe 2 enhancer (eveS2) fails to drive appreciable expression in D. melanogaster [1]. However, we found that a large transgene carrying the entire $D$. erecta eve locus drives normal eve expression, including in stripe 2. We performed a functional dissection of the region upstream of the $D$. erecta eveS2 region and found that regulatory information outside of the minimal $D$. erecta eveS2 contains multiple Zelda motifs that are required for normal expression. Our results illustrate how sequences outside of minimal enhancer regions can evolve functionally through mechanisms other than changes in transcription factor binding sites that drive patterning.
\end{abstract}

\section{Keywords}

Evolution - Transcription - Enhancer - Drosophila - Even-skipped

1 Janelia Research Campus, Howard Hughes Medical Institute, 19700 Helix Drive, Ashburn, VA 20147 USA

*Corresponding author:crockerj@janelia.hhmi.org

\section{Introduction}

Developmental enhancers contain multiple binding sites for transcription factors, together specifying the precise time, level, and location of gene expression. Classically, minimal enhancers have been identified as the smallest DNA fragments that are sufficient to direct reporter-gene expression in a particular tissue or domain of normal gene expression $[2,3]$. These studies have provided mechanistic insight into how transcriptional logic is encoded in individual enhancers $[4,5]$. However, in even the earliest studies, it was clear that minimal enhancers are insufficient to define the normal gene expression pattern with complete fidelity [2]. More recently, genomic studies have provided evidence that minimal enhancers are embedded within larger regions containing additional transcription factor binding sites that may be required for normal enhancer function $[6,7,8]$.

Phenotypic evolution results largely from sequence changes in enhancers $[9,10,11,12,13]$, even between closely related species $[14,15,16,17,18,19]$. It is not clear, however, how often functional evolution includes changes within minimal enhancers versus outside of these regions. We have explored this problem through studies of the even-skipped (eve) gene.

The Drosophila melanogaster eve gene is expressed in seven transverse stripes along the anterior-posterior axis in the blastoderm embryo [20, 21]. Minimal enhancers have been identified that each direct expression in either one or two stripes that together drive expression in all seven stripes
$[22,23]$. Of all these enhancers, the minimal element for stripe 2 has been studied in the greatest detail [2, 24, 25]. This enhancer contains multiple binding sites for transcriptional activators (Bicoid and Hunchback) and repressors (Giant, Krüppel, and Sloppy-paired). The collective activity of transcription factors binding to these sites drives eve expression specifically in stripe 2 [2, 21, 24, 25, 26].

Previously, reporter gene assays were used to investigate the functional evolution of eve $S 2$ from three divergent Drosophila species with transgenic assays in D. melanogaster $[1,27,15,28,6,29]$. These studies revealed that the eveS2 enhancers from D. yakuba and D. pseudoobscura, which diverged $\sim 10$ and $\sim 40$ million years ago, respectively, from $D$. melanogaster, drove apparently normal expression in stripe 2 . However, eveS2 from $D$. erecta, which is closely related to $D$. yakuba, failed to drive appreciable levels of expression.

There are several possible reasons for why the D. erecta eveS2 element does not drive appreciable expression in $D$. melanogaster [1]. First, the "minimal" functional D. erecta eveS2 element may have been replaced elsewhere in the $D$. erecta eve locus with a functionally equivalent enhancer. Second, the D. erecta enhancer may contain all the required information to drive native expression, but the enhancer may have evolved to accommodate differences in the $D$. erecta embryonic environment [30,31]—for example, differences in transcription factor concentrations [17, 32]. Third, the true functional eveS 2 enhancer may be larger than defined by the D. melanogaster minimal element and shifts in the locations 
bioRxiv preprint doi: https://doi.org/10.1101/101311; this version posted January 18,2017 . The copyright holder for this preprint (which was not certified by peer review) is the author/funder, who has granted bioRxiv a license to display the preprint in perpetuity. It is made available under aCC-BY-NC 4.0 International license.

Functional regulatory evolution outside of the minimal even-skipped stripe 2 enhancer - 2/12

of key transcription factor binding sites may have rendered the $D$. erecta region corresponding to the $D$. melanogaster "minimal" element unable to drive appropriate expression.

Here we tested these hypotheses with a functional dissection of the $D$. erecta eve locus in transgenic D. melanogaster. We found that a large transgene carrying the entire $D$. erecta eve locus drove normal expression in all seven stripes. This $D$. erecta transgene rescued down-stream eve targets and larval segmentation defects caused by an eve null mutation. We found that regulatory information required for $D$. erecta stripe 2 expression is located outside of the minimal stripe 2 enhancer region. Finally, we found that the $D$. erecta minimal eveS2 region lacks multiple Zelda transcription factor binding sites that are found in D. melanogaster and we demonstrate that normal function of the $D$. erecta eveS2 enhancer requires Zelda transcription factor binding motifs located outside of the minimal enhancer element. Zelda is apparently not required for patterning, but instead for making enhancers accessible for regulation [33, 34, 35, 36, 37, 38, 39, 40]. Many studies have suggested that the transcription factor binding sites required for normal enhancer function are often distributed over regions larger than experimentally-determined minimal enhancer elements and our results demonstrate that critical transcription factor binding sites can shift during evolution between locations within and outside of "minimal" regions.

\section{Results}

D. melanogaster and D. erecta both express eve in seven embryonic stripes at similar levels and locations (Figure 1 a, b). A transgene of the D. melanogaster eveS2 minimal enhancer, when re-introduced into D. melanogaster, drives robust expression in approximately the same region as the native expression of eve stripe 2 (Figure 1c). However, the orthologous eve $S 2$ fragment from $D$. erecta does not drive expression in transgenic D. melanogaster (Figure 1d) (see also Ludwig et al., [1]). A $20 \mathrm{~kb}$ region of the D. melanogaster genome surrounding the eve transcription unit is sufficient to drive apparently normal eve expression and rescues transcription of genes that are normally regulated by Eve [23]. To maximize the likelihood that we would capture the entire eve locus from $D$. erecta, we tested the ability of a $\sim 47 \mathrm{~kb}$ $D$. erecta fosmid to drive expression in D. melanogaster embryos deficient for native eve function. This fosmid contains D. erecta sequences orthologous to all of the D. melanogaster stripe enhancers (Fig. 1e).

We found that this $D$. erecta fosmid drove apparently normal expression of all seven eve stripes (Fig. 1f-k). To determine whether the timing, levels, and spatial distribution of Eve expression driven by the D. erecta fosmid were correct in D. melanogaster, we tested the ability of the D. erecta locus to functionally rescue gene regulatory networks that are normally regulated by Eve. We found that the $D$. erecta eve fosmid rescued the expression of the segmental polarity gene engrailed (Fig. 2a-c). Additionally, we found that the fosmid rescued all segmentation defects in the cuticles of
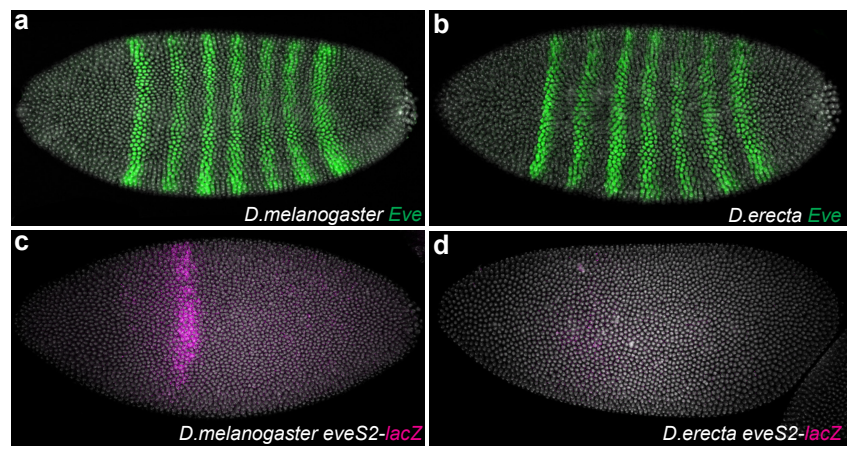

e

D. erecta eve Fosmid
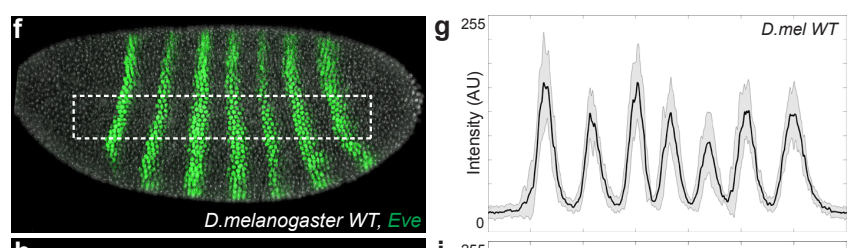

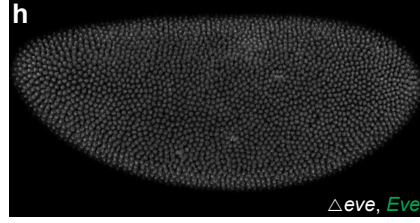
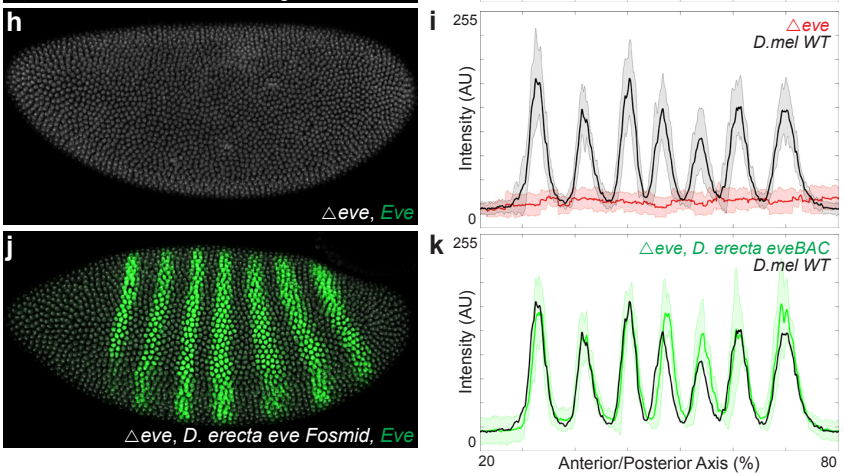

Figure 1. The $D$. erecta eve locus drives normal eve expression in transgenic $D$. melanogaster. $(\mathbf{a}, \mathbf{b})$ Stage 5 embryos stained for Eve protein in either D. melanogaster (a) or D. erecta (b). (c, d) Stage 5 D. melanogaster embryos stained for $B-G a l$ RNA carrying the $D$. melanogaster eveS2 (c) or $D$. erecta eveS2. e) Schematic representation of the eve locus with enhancers indicated in dark boxes. (f, h, j) Stage 5 embryos stained for Eve protein in either a wild-type background (f), eve null background (h), or an eve null background carrying the $D$. erecta eve fosmid (j). ( $\mathbf{g}, \mathbf{i}, \mathbf{k})$ Profiles of average expression levels across the region indicated in panel $(\mathbf{f})$ for the indicated genotype $(n=10$ for each genotype). In all plots, the solid black line denotes wild-type, red denotes eve null (i), and green denotes an eve null background carrying the $D$. erecta eve fosmid (k). Bounding areas around experimental data indicate one standard deviation. AU indicates Arbitrary Units of fluorescence intensity.

larvae deficient for eve (Fig. 2d-f). Together, these results suggest that the $47 \mathrm{~kb} D$. erecta fosmid contains all of the regulatory information required for eve expression in stripe 2 and all other eve stripes.

The regulatory information for $D$. melanogaster eve stripe 2 and stripes 3/7 is located upstream of the eve promoter $[41,2,25,42]$. Increasing the size of the DNA regions tested for these enhancers increases levels of transcription [41, 2, 
bioRxiv preprint doi: https://doi.org/10.1101/101311; this version posted January 18,2017 . The copyright holder for this preprint (which was not certified by peer review) is the author/funder, who has granted bioRxiv a license to display the preprint in perpetuity. It is made available under aCC-BY-NC 4.0 International license.

Functional regulatory evolution outside of the minimal even-skipped stripe 2 enhancer $-3 / 12$
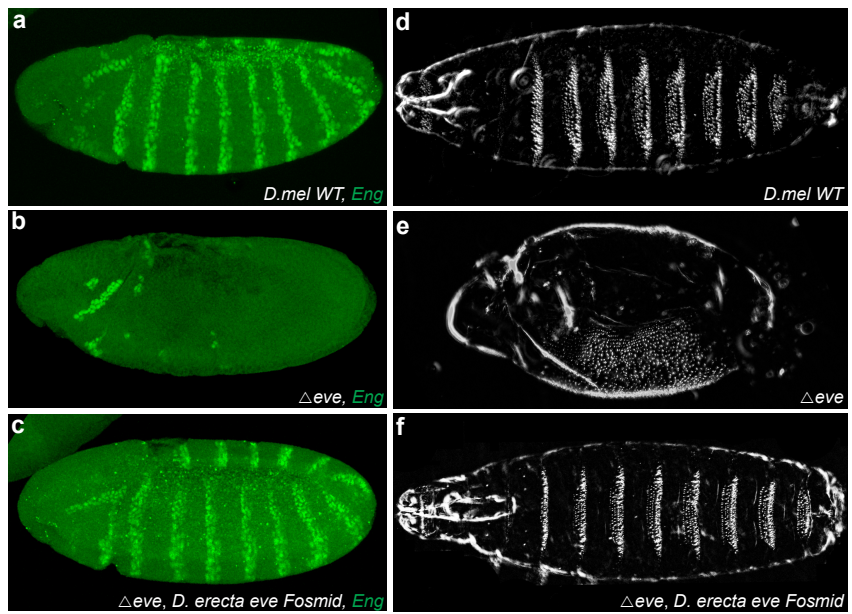

Figure 2. Functional rescue of eve null flies by the $D$. erecta fosmid. (a, b, c) Stage 9 embryos stained for Engrailed (Eng) protein in either a wild-type background (a), eve null background (b), or an eve null background carrying the $D$. erecta eve fosmid (c). (d, e, f) First instar larval cuticle preps of either a wild-type (d), eve null background (e), or an eve null background carrying the $D$. erecta eve fosmid (f).

25, 42]. Additionally, while the minimal D. melanogaster enhancer is sufficient to drive approximately normal expression of eve stripe 2, sequences surrounding the minimal eveS2 element contribute to the robustness of the minimal enhancer [6]. Therefore, we suspected that sequences near the minimal D. erecta eve $S 2$ may be required for normal expression.

To test if regulatory information $5^{\prime}$ of the minimal $D$. erecta eveS 2 region is required for normal stripe 2 expression, we tested a series of constructs that included the minimal stripe 2 enhancer plus progressively more 5' DNA (Fig. 3a). While the 855 bp D. erecta minimal eveS2 construct drove very little expression in D. melanogaster (Fig. 3b, c), a fragment containing an additional $832 \mathrm{bp}$ upstream of the minimal enhancer drove weak expression in the stripe two domain (Fig. $3 \mathrm{~d}$, e). Increasing the minimal construct size with an additional $1609 \mathrm{bp} 5$ ' of the minimal enhancer, up to the boundary with the minimal stripe 3/7 enhancer, further increased levels of stripe 2 expression and drove weak expression in stripes 3 and 7 (Fig. $3 \mathrm{f}, \mathrm{g}$ ). This indicates both that information critical for stripe 2 expression resides outside the minimal stripe 2 region in $D$. erecta, but also that patterning information for stripes 3 and 7 resides outside of the minimal stripe $3 / 7$ enhancer. Increasing the size of the element further, so that it encompassed the minimal eve $3 / 7$ enhancer, did not further increase expression levels in the domain of eve stripe two, but increased expression in stripes 3 and 7 (Fig. 3h, i). Together, these results demonstrate that up to $1609 \mathrm{bp} 5$ ' of the minimal $D$. erecta eve stripe-two enhancer contains regulatory information required for eve expression in transgenic D. melanogaster.

We next performed a computational search for binding sites of three transcription factors that regulate the spatial pat-

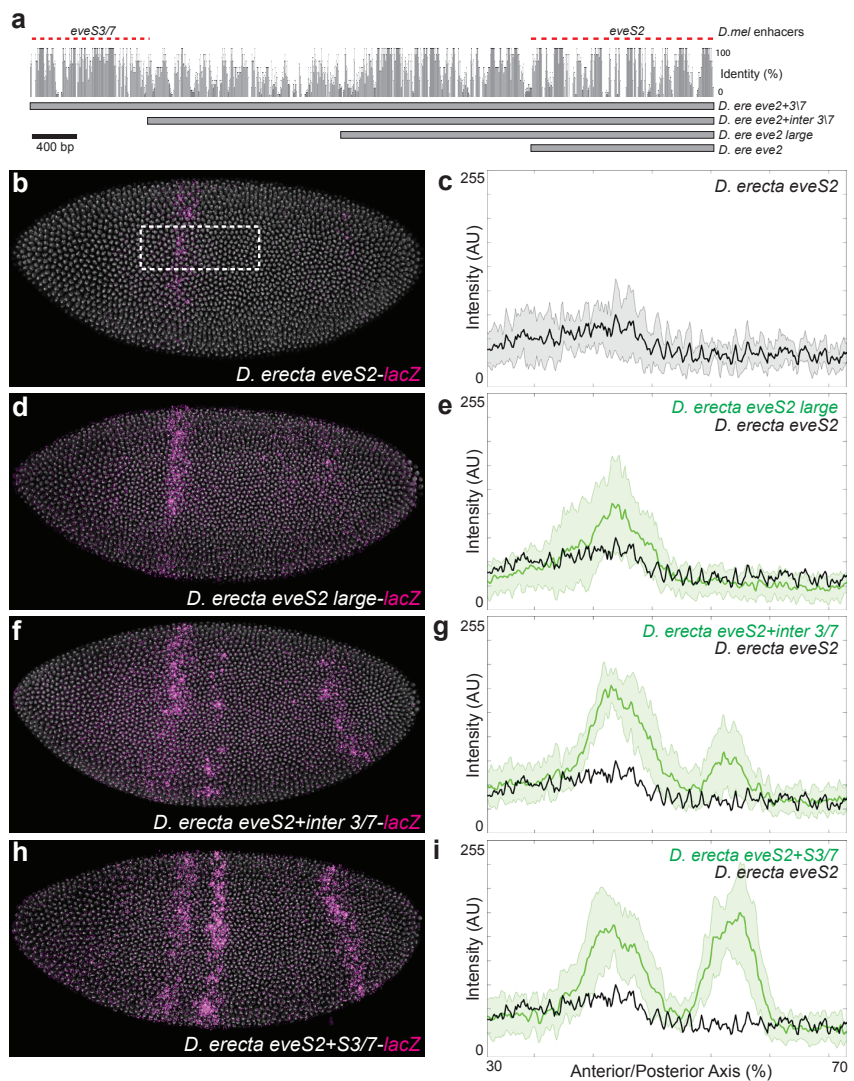

Figure 3. Sequences upstream of the minimal $D$. erecta eveS2 enhancer drive expression in $D$. melanogaster. (a) Schematic representation of the eve locus with enhancers tested indicated. The sequence conservation plot is a 5-way sequence alignment between $D$. melanogaster, sechellia, yakuba, erecta, and ananassae. (b, d, f, h) Stage $5 D$. melanogaster embryos stained for $B-G a l$ RNA carrying the indicated transgene. (c, e, g, i) Profiles of average expression levels across the region indicated in panel (b) for the indicated enhancer ( $n=10$ for each genotype). In all plots, the solid black line denotes wild-type, and green denotes the $D$. erecta eve enhancer (k). Bounding areas around experimental data indicate one standard deviation. AU indicates Arbitrary Units of fluorescence intensity.

tern of the D. melanogaster eveS2 enhancer-Bicoid, Hunchback and Krüppel-across the regulatory region upstream of the minimal enhancer (Fig. S1). We observed no obvious turnover of these binding sites in D. erecta that would explain the loss of expression from the minimal eveS2 enhancer, consistent with previous studies [1].

We next examined the spatial distribution of putative binding sites for the Zelda protein. Zelda is expressed ubiquitously in the blastoderm embryo and Zelda protein binds to many enhancers that drive transcription in the early blastoderm embryo $[33,34,35,36,37,38,39,40]$. Zelda activity is correlated with chromatin accessibility [35, 39, 40] and Zelda appears to make enhancers accessible to transcription factors that drive specific patterns of gene expression [34, 35, 38, 39, 40, 43].

We searched for putative Zelda motifs in the eveS2 mini- 
bioRxiv preprint doi: https://doi.org/10.1101/101311; this version posted January 18,2017 . The copyright holder for this preprint (which was not certified by peer review) is the author/funder, who has granted bioRxiv a license to display the preprint in perpetuity. It is made available under aCC-BY-NC 4.0 International license.

Functional regulatory evolution outside of the minimal even-skipped stripe 2 enhancer $-4 / 12$

mal element in D. melanogaster, D. sechellia, D. yakuba, and $D$. erecta. We found four sites that were perfectly conserved between these species, and six sites that were present in $D$. melanogaster but absent in $D$. erecta. It is possible that the loss of these motifs has led to the loss of activity of the $D$. erecta eve 2 minimal element. Because the larger $D$. erecta reporter constructs we tested drove stripe 2 expression, we searched for additional Zelda motifs 5' of the minimal element. We identified three Zelda motifs in this region in $D$. erecta, two of which are conserved in D. melanogaster and one of which is absent in D. melanogaster (Fig. 4a, b). We tested the activity of these motifs in vivo by deleting all three motifs from the large D. erecta eveS 2 construct (Fig. $4 \mathrm{c}, \mathrm{d}$ ). Removal of these upstream Zelda motifs abrogated reporter gene expression (Fig. 4d). These Zelda motifs are therefore critical for stripe 2 expression in $D$. erecta.

To test if these Zelda motifs were sufficient to drive expression when appended to the minimal $D$. erecta eve $S 2$, we placed the three Zelda motifs upstream of the minimal element. Strikingly, we found that this construct drove expression in stripe 2 (Fig. 4e, f). Addition of these Zelda sites is therefore sufficient to allow properly patterned expression of the minimal $D$. erecta stripe 2 element. Furthermore, the patterns of Zelda motif gain and loss suggest that loss of Zelda motifs within the minimal elements prevents the $D$. erecta minimal enhancer element from driving expression and that gain of a new Zelda motif upstream of the minimal element may be required for normal $D$. erecta stripe 2 expression.

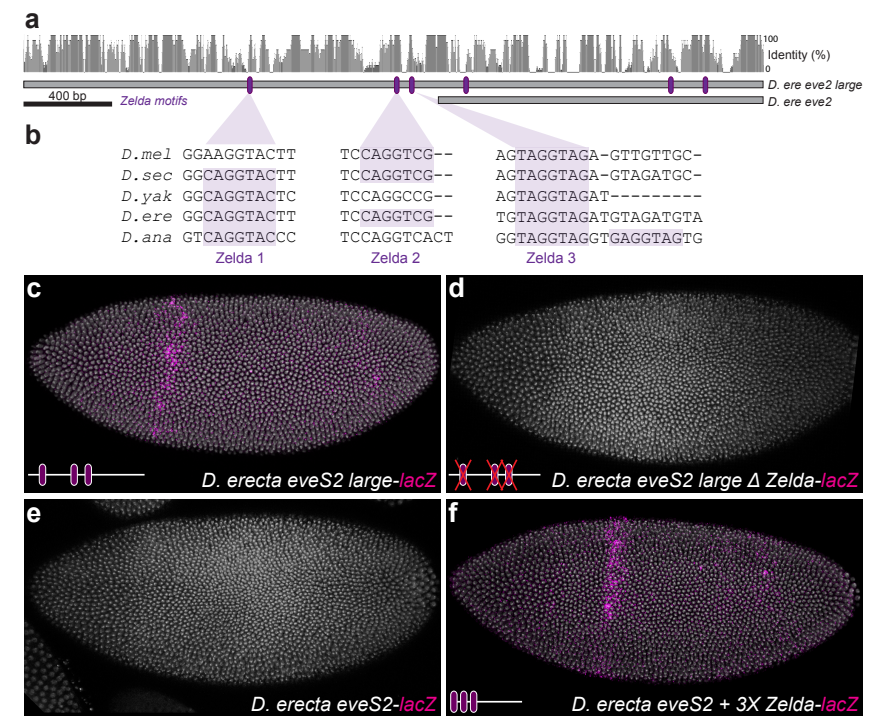

Figure 4. Zelda motifs are necessary and sufficient for the expression of the minimal $D$. erecta eveS2. $(\mathbf{a}, \mathbf{b})$ Schematic representation of the eve locus, with tested enhancers indicated, highlighting the upstream Zelda motifs in $D$. erecta (b). The sequence conservation plot is a 5-way sequence alignment between $D$. melanogaster, sechellia, yakuba, erecta, and ananassae. (c, d, e, f) Stage $5 D$. melanogaster embryos stained for $B$-Gal RNA carrying the indicated $D$. erecta transgene

\section{Discussion}

Our results suggest that functional regulatory evolution has occurred outside the minimal eveS2 enhancer between $D$. melanogaster and D. erecta. The minimal D. erecta eveS2 region contains binding sites necessary for patterning of expression in stripe 2, but lacks sufficient Zelda binding sites to drive expression. These results support the hypothesis that Zelda contributes to determining the regulatory state of the eveS2 enhancer in the blastoderm embryo, ON versus OFF, and can be decoupled, at least in part, from the patterns and levels of expression driven by the enhancer [34, 35, 38, 39, 40, 43]. We have not explored the $777 \mathrm{bp}$ upstream of these Zelda sites (the extra DNA in construct $D$. ere eve $2+$ inter $3 \backslash 7$ ) for the sequences that drive stronger expression in stripe 2 . This is a poorly conserved genomic region and it is curious that it contains information both for bolstering stripe 2 expression and for patterning stripes 3 and 7 . The function of this DNA region would repay further exploration.

This study highlights the need to understand gene regulatory evolution at the level of the entire locus. While studies of minimal enhancers elements have provided enormous insight into the mechanisms of gene regulation, the broader DNA regions around minimal enhancers also contribute to enhancer function and evolution [44, 45, 46, 47, 48, 49, 50]. Our results encourage great caution in comparative studies of enhancer evolution when functional studies have been performed on enhancers from only one species. One goal for future research is to understand how gene regulation evolves at the level of the entire locus, including information such as binding site composition, the arrangement of enhancers, the activities of intervening sequences, and the distance between functional sequences.

\section{Methods and Materials}

\subsection{Construction of enhancer constructs.}

The $D$. erecta locus was tested by cloning the BDERF014213 fosmid, obtained from BACPAC resources (https:// bacpacresources.org/), corresponding to D. melanogaster chr2R:5,840,325-5,887,364, into vector TKBL-w+ [51]. All enhancer constructs were cloned into the placZattB expression construct with a hsp70 promoter [18]. See supplemental materials for complete construct sequences.

\subsection{Fly strains and crosses.}

D. melanogaster strains were maintained under standard laboratory conditions. Transgenic enhancer constructs were created by Rainbow Transgenic Flies Inc. and were integrated at the attP2 landing site. The eveR13 mutation was used to test for complementation using the $D$. erecta fosmid. The lethal mutations were balanced over marked balancer chromosome $\mathrm{CyO} P($ hb-lacZ) to allow identification of mutant embryos by immunostaining for Beta-galactosidase. 


\subsection{Embryo manipulations.}

Embryos were raised at $25^{\circ} \mathrm{C}$ and fixed and stained according to standard protocols [52]. Briefly, primary antibodies obtained from the Developmental Studies Hybridoma Bank were used to detect Eve (3C10, used 1:20) and En (4D9, used $1: 20)$ proteins, which was followed by detection of primary antibodies using secondary antibodies labeled with Alexa Fluor dyes (1:500, Invitrogen). Cuticle preps were performed using standard protocols [53].

\subsection{Microscopy.}

Each series of experiments to measure transcript levels was performed entirely in parallel. Embryo collections, fixations, and hybridizations, image acquisitions, and processing were performed side-by-side in identical conditions. Confocal exposures were identical for each series and were set to not exceed the 255 maximum level. Confocal images were obtained on a Leica DM5500 Q Microscope with an ACS APO $20 \times / 0.60$ IMM CORR lens and Leica Microsystems LAS AP software. Sum projections of confocal stacks were assembled, embryos were scaled to match sizes, background was subtracted using a 50-pixel rolling-ball radius and plot profiles of fluorescence intensity were analyzed using ImageJ software (http://rsb.info.nih.gov/ij/). Data from the plot profiles were further analyzed in Matlab.

\section{Acknowledgments}

We thank the entire Stern lab for discussion and comments, Claire Standley for comments on the manuscript, and Jessica Cande for the Bourbon fruit cake that proved important for manuscript preparation.

\section{References}

[1] Michael Z Ludwig, Arnar Palsson, Elena Alekseeva, Casey M Bergman, Janaki Nathan, and Martin Kreitman. Functional evolution of a cis-regulatory module. PLoS biology, 3(4):e93, apr 2005.

[2] S Small, A Blair, M Levine, S Small, and M Levine. Regulation of even-skipped stripe 2 in the Drosophila embryo. The EMBO journal, 11(11):4047-57, nov 1992.

[3] Justin Crocker and David L Stern. TALE-mediated modulation of transcriptional enhancers in vivo. Nature methods, 10(8):762-7, aug 2013.

[4] François Spitz and Eileen E M Furlong. Transcription factors: from enhancer binding to developmental control. Nature reviews. Genetics, 13(9):613-26, sep 2012.

[5] Michal Levo and Eran Segal. In pursuit of design principles of regulatory sequences. Nature reviews. Genetics, 15(7):453-68, jul 2014.

[6] Michael Z Ludwig, Manu, Ralf Kittler, Kevin P White, and Martin Kreitman. Consequences of eukaryotic enhancer architecture for gene expression dynamics, devel- opment, and fitness. PLoS genetics, 7(11):e1002364, nov 2011.

[7] Cosmas D Arnold, Daniel Gerlach, Daniel Spies, Jessica A Matts, Yuliya A Sytnikova, Michaela Pagani, Nelson C Lau, and Alexander Stark. Quantitative genomewide enhancer activity maps for five Drosophila species show functional enhancer conservation and turnover during cis-regulatory evolution. Nature Genetics, 46(7):685692, jun 2014.

[8] David S Lorberbaum, Andrea I Ramos, Kevin A Peterson, Brandon S Carpenter, David S Parker, Sandip De, Lauren E Hillers, Victoria M Blake, Yuichi Nishi, Matthew R McFarlane, Ason CY Chiang, Judith A Kassis, Benjamin L Allen, Andrew P McMahon, and Scott Barolo. An ancient yet flexible $<\mathrm{i}>$ cis $-</ \mathrm{i}>$ regulatory architecture allows localized Hedgehog tuning by $<\mathrm{i}>$ patched/Ptch $1<\mathrm{i}>$. eLife, 5, may 2016.

[9] Mike Levine. Transcriptional enhancers in animal development and evolution. Current biology : $C B$, 20(17):R754-63, sep 2010.

[10] Sara L. Prescott, Rajini Srinivasan, Maria Carolina Marchetto, Irina Grishina, Iñigo Narvaiza, Licia Selleri, Fred H. Gage, Tomek Swigut, and Joanna Wysocka. Enhancer Divergence and cis-Regulatory Evolution in the Human and Chimp Neural Crest. Cell, sep 2015.

[11] William J. Glassford, Winslow C. Johnson, Natalie R. Dall, Sarah Jacquelyn Smith, Yang Liu, Werner Boll, Markus Noll, and Mark Rebeiz. Co-option of an Ancestral Hox-Regulated Network Underlies a Recently Evolved Morphological Novelty. Developmental Cell, 34(5):520-531, sep 2015.

[12] Ella Preger-Ben Noon, Fred P. Davis, and David L. Stern. Evolved Repression Overcomes Enhancer Robustness. Developmental Cell, 39(5):572-584, dec 2016.

[13] Evgeny Z. Kvon, Olga K. Kamneva, Uirá S. Melo, Iros Barozzi, Marco Osterwalder, Brandon J. Mannion, Virginie Tissières, Catherine S. Pickle, Ingrid Plajzer-Frick, Elizabeth A. Lee, Momoe Kato, Tyler H. Garvin, Jennifer A. Akiyama, Veena Afzal, Javier Lopez-Rios, Edward M. Rubin, Diane E. Dickel, Len A. Pennacchio, and Axel Visel. Progressive Loss of Function in a Limb Enhancer during Snake Evolution. Cell, 167(3):633642.e11, oct 2016.

[14] Stewart MacArthur and John F Y Brookfield. Expected rates and modes of evolution of enhancer sequences. Molecular biology and evolution, 21(6):1064-73, 2004.

[15] M Z Ludwig and M Kreitman. Evolutionary dynamics of the enhancer region of even-skipped in Drosophila. Molecular biology and evolution, 12(6):1002-11, nov 1995.

[16] Alex S Nord, Matthew J Blow, Catia Attanasio, Jennifer A Akiyama, Amy Holt, Roya Hosseini, Sengthavy 
Phouanenavong, Ingrid Plajzer-Frick, Malak Shoukry, Veena Afzal, John L R Rubenstein, Edward M Rubin, Len A Pennacchio, and Axel Visel. Rapid and pervasive changes in genome-wide enhancer usage during mammalian development. Cell, 155(7):1521-31, dec 2013.

[17] Justin Crocker, Yoichiro Tamori, and Albert Erives. Evolution acts on enhancer organization to fine-tune gradient threshold readouts. PLoS biology, 6(11):e263, nov 2008.

[18] Justin Crocker, Namiko Abe, Lucrezia Rinaldi, Alistair P McGregor, Nicolás Frankel, Shu Wang, Ahmad Alsawadi, Philippe Valenti, Serge Plaza, François Payre, Richard S Mann, and David L Stern. Low affinity binding site clusters confer hox specificity and regulatory robustness. Cell, 160(1-2):191-203, jan 2015.

[19] Nicolás Frankel, Deniz F Erezyilmaz, Alistair P McGregor, Shu Wang, François Payre, and David L Stern. Morphological evolution caused by many subtle-effect substitutions in regulatory DNA. Nature, 474(7353):598-603, jun 2011.

[20] P M Macdonald, P Ingham, and G Struhl. Isolation, structure, and expression of even-skipped: a second pairrule gene of Drosophila containing a homeo box. Cell, 47(5):721-34, dec 1986.

[21] Garth R Ilsley, Jasmin Fisher, Rolf Apweiler, Angela H Depace, and Nicholas M Luscombe. Cellular resolution models for even skipped regulation in the entire Drosophila embryo. eLife, 2:e00522, jan 2013.

[22] S Small, D N Arnosti, and M Levine. Spacing ensures autonomous expression of different stripe enhancers in the even-skipped promoter. Development (Cambridge, England), 119(3):762-72, 1993.

[23] M Fujioka, Y Emi-Sarker, G L Yusibova, T Goto, and J B Jaynes. Analysis of an even-skipped rescue transgene reveals both composite and discrete neuronal and early blastoderm enhancers, and multi-stripe positioning by gap gene repressor gradients. Development (Cambridge, England), 126(11):2527-38, jun 1999.

[24] D Stanojevic, S Small, and M Levine. Regulation of a segmentation stripe by overlapping activators and repressors in the Drosophila embryo. Science (New York, N.Y.), 254(5036):1385-7, nov 1991.

[25] S Small, R Warrior, R Kraut, M Levine, T Hoey, R Warrior, and $\mathrm{M}$ Levine. Transcriptional regulation of a pairrule stripe in Drosophila. Genes \& development, 5(5):82739, may 1991.

[26] Justin Crocker, Garth R Ilsley, and David L Stern. Quantitatively predictable control of Drosophila transcriptional enhancers in vivo with engineered transcription factors. Nature genetics, 48(3):292-8, mar 2016.

[27] M Z Ludwig, N H Patel, and M Kreitman. Functional analysis of eve stripe 2 enhancer evolution in Drosophila: rules governing conservation and change. Development, 125(5):949-958, mar 1998.

[28] M Z Ludwig, C Bergman, N H Patel, and M Kreitman. Evidence for stabilizing selection in a eukaryotic enhancer element. Nature, 403(6769):564-7, feb 2000.

[29] C. Martinez, J. S. Rest, A.-R. Kim, M. Ludwig, M. Kreitman, K. White, and J. Reinitz. Ancestral Resurrection of the Drosophila S2E Enhancer Reveals Accessible Evolutionary Paths through Compensatory Change. Molecular Biology and Evolution, 31(4):903-916, apr 2014.

[30] Charless C Fowlkes, Kelly B. Eckenrode, Meghan D. Bragdon, Miriah Meyer, Zeba Wunderlich, Lisa Simirenko, Cris L. Luengo Hendriks, Soile V E Keränen, Clara Henriquez, David W Knowles, Mark D Biggin, Michael B Eisen, and Angela H. DePace. A Conserved Developmental Patterning Network Produces Quantitatively Different Output in Multiple Species of Drosophila. PLoS Genetics, 7(10):e1002346, oct 2011.

[31] Antoine Barrière, Kacy L. Gordon, and Ilya Ruvinsky. Coevolution within and between Regulatory Loci Can Preserve Promoter Function Despite Evolutionary Rate Acceleration. PLoS Genetics, 8(9):e1002961, sep 2012.

[32] Juan Sebastian Chahda, Rui Sousa-Neves, and Claudia Mieko Mizutani. Variation in the Dorsal Gradient Distribution Is a Source for Modified Scaling of Germ Layers in Drosophila. Current Biology, 23(8):710-716, apr 2013.

[33] Hsiao-lan Liang, Chung-yi Nien, Hsiao-yun Liu, Mark M Metzstein, Nikolai Kirov, and Christine Rushlow. The zinc-finger protein Zelda is a key activator of the early zygotic genome in Drosophila. Nature, 456(November):400-404, 2008.

[34] Zhe Xu, Hongtao Chen, Jia Ling, Danyang Yu, Paolo Struffi, and Stephen Small. Impacts of the ubiquitous factor Zelda on Bicoid-dependent DNA binding and transcription in Drosophila. Genes \& development, 28(6):60821, mar 2014.

[35] Sun Melody Foo, Yujia Sun, Bomyi Lim, Ruta Ziukaite, Kevin O'Brien, Chung-Yi Nien, Nikolai Kirov, Stanislav Y Shvartsman, and Christine A Rushlow. Zelda potentiates morphogen activity by increasing chromatin accessibility. Current biology : CB, 24(12):1341-6, jun 2014.

[36] Melissa M Harrison, Xiao-Yong Li, Tommy Kaplan, Michael R Botchan, and Michael B Eisen. Zelda binding in the early Drosophila melanogaster embryo marks regions subsequently activated at the maternal-to-zygotic transition. PLoS genetics, 7(10):e1002266, oct 2011.

[37] Chung-Yi Nien, Hsiao-Lan Liang, Stephen Butcher, Yujia Sun, Shengbo Fu, Tenzin Gocha, Nikolai Kirov, J Robert Manak, and Christine Rushlow. Temporal coordination of gene networks by Zelda in the early Drosophila embryo. PLoS genetics, 7(10):e1002339, oct 2011. 
bioRxiv preprint doi: https://doi.org/10.1101/101311; this version posted January 18,2017 . The copyright holder for this preprint (which was not certified by peer review) is the author/funder, who has granted bioRxiv a license to display the preprint in perpetuity. It is made available under aCC-BY-NC 4.0 International license.

Functional regulatory evolution outside of the minimal even-skipped stripe 2 enhancer - 7/12

[38] Xiao-Yong Li, Melissa M Harrison, Jacqueline E Villalta, Tommy Kaplan, and Michael B Eisen. Establishment of regions of genomic activity during the Drosophila maternal to zygotic transition. eLife, 3, jan 2014.

[39] Yujia Sun, Chung-Yi Nien, Kai Chen, Hsiao-Yun Liu, Jeff Johnston, Julia Zeitlinger, and Christine Rushlow. Zelda overcomes the high intrinsic nucleosome barrier at enhancers during Drosophila zygotic genome activation. Genome research, sep 2015.

[40] Katharine N Schulz, Eliana R Bondra, Arbel Moshe, Jacqueline E Villalta, Jason D Lieb, Tommy Kaplan, Daniel J McKay, and Melissa M Harrison. Zelda is differentially required for chromatin accessibility, transcription-factor binding and gene expression in the early Drosophila embryo. Genome research, sep 2015.

[41] D N Arnosti, S Barolo, M Levine, and S Small. The eve stripe 2 enhancer employs multiple modes of transcriptional synergy. Development (Cambridge, England), 122(1):205-14, 1996.

[42] S Small, A Blair, and M Levine. Regulation of two pair-rule stripes by a single enhancer in the Drosophila embryo. Developmental biology, 175(2):314-24, 1996.

[43] Justin Crocker, Albert Tsai, and David L. Stern. A Fully Synthetic Transcriptional Platform for a Multicellular Eukaryote. Cell Reports, 18(1):287-296, jan 2017.

[44] Wouter de Laat and Denis Duboule. Topology of mammalian developmental enhancers and their regulatory landscapes. Nature, 502(7472):499-506, oct 2013.

[45] Orsolya Symmons, Leslie Pan, Silvia Remeseiro, Tugce Aktas, Felix Klein, Wolfgang Huber, and François Spitz. The Shh Topological Domain Facilitates the Action of Remote Enhancers by Reducing the Effects of Genomic Distances. Developmental Cell, 39(5):529-543, dec 2016.

[46] Hinco J Gierman, Mireille H G Indemans, Jan Koster, Sandra Goetze, Jurgen Seppen, Dirk Geerts, Roel van Driel, and Rogier Versteeg. Domain-wide regulation of gene expression in the human genome. Genome research, 17(9):1286-95, sep 2007.

[47] Waseem Akhtar, Johann de Jong, Alexey V. V Pindyurin, Ludo Pagie, Wouter Meuleman, Jeroen de Ridder, Anton Berns, Lodewyk F.A. F A Wessels, Maarten van Lohuizen, Bas van Steensel, Johann de Jong, Alexey V. V Pindyurin, Ludo Pagie, Wouter Meuleman, Jeroen de Ridder, Anton Berns, Lodewyk F.A. F A Wessels, Maarten van Lohuizen, and Bas van Steensel. Chromatin position effects assayed by thousands of reporters integrated in parallel. Cell, 154(4):914-27, aug 2013.

[48] Menzies Chen, Katherine Licon, Rei Otsuka, Lorraine Pillus, and Trey Ideker. Decoupling Epigenetic and Genetic Effects through Systematic Analysis of Gene Position. Cell Reports, 3(1):128-137, 2013.
[49] Xiaoshu Chen and Jianzhi Zhang. The Genomic Landscape of Position Effects on Protein Expression Level and Noise in Yeast. 2016.

[50] Leslie Dunipace, Anil Ozdemir, and Angelike Stathopoulos. Complex interactions between cis-regulatory modules in native conformation are critical for Drosophila snail expression. Development, 138(18), 2011.

[51] Shu Kondo, Matthew Booker, and Norbert Perrimon. Cross-species RNAi rescue platform in Drosophila melanogaster. Genetics, 183(3):1165-73, nov 2009.

[52] Justin Crocker and Albert Erives. A Schnurri/Mad/Medea complex attenuates the dorsal-twist gradient readout at vnd. Developmental biology, 378(1):64-72, jun 2013.

[53] David L Stern and Elio Sucena. Preparation of cuticles from feeding Drosophila larvae. Cold Spring Harbor protocols, 2011(11):1394-8, nov 2011.

[54] Gabriela G Loots, Ivan Ovcharenko, Lior Pachter, Inna Dubchak, and Edward M Rubin. rVista for comparative sequence-based discovery of functional transcription factor binding sites. Genome research, 12(5):832-9, may 2002.

[55] Alexander P Lifanov, Vsevolod J Makeev, Anna G Nazina, and Dmitri A Papatsenko. Homotypic regulatory clusters in Drosophila. Genome research, 13(4):579-88, apr 2003.

[56] Justin Crocker, Nathan Potter, and Albert Erives. Dynamic evolution of precise regulatory encodings creates the clustered site signature of enhancers. Nature communications, 1(7):99, jan 2010. 
bioRxiv preprint doi: https://doi.org/10.1101/101311; this version posted January 18,2017 . The copyright holder for this preprint (which was not certified by peer review) is the author/funder, who has granted bioRxiv a license to display the preprint in perpetuity. It is made available under aCC-BY-NC 4.0 International license.

Functional regulatory evolution outside of the minimal even-skipped stripe 2 enhancer $-8 / 12$

\section{Appendices}

\section{Supplemental Figures}
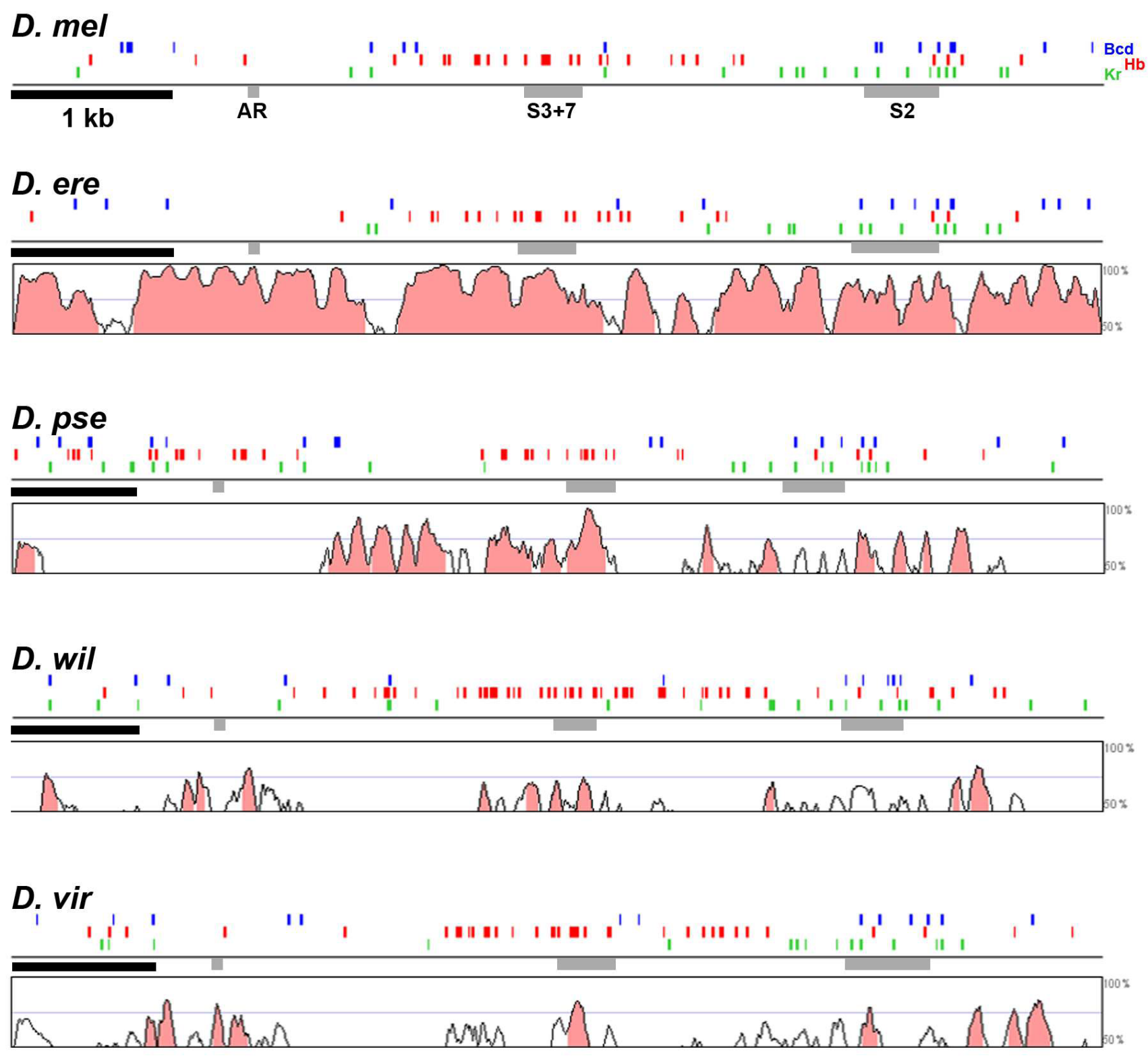

Figure S1. VISTA plot demonstrating extensive sequence conservation upstream of the eve start site [54]. Gray boxes denote regions homologous to the $D$. melanogaster minimal regulatory elements. Computationally predicted binding sites based on reported position-weighted matrices [55], demonstrate an extensive number of conserved binding sites for Bcd (Blue), Hb (Red), and $\mathrm{Kr}$ (Green) 
bioRxiv preprint doi: https://doi.org/10.1101/101311; this version posted January 18,2017 . The copyright holder for this preprint (which was not certified by peer review) is the author/funder, who has granted bioRxiv a license to display the preprint in perpetuity. It is made available under

Functional regulatory evolution outside of the minimal even-skipped stripe 2 enhancer - 9/12

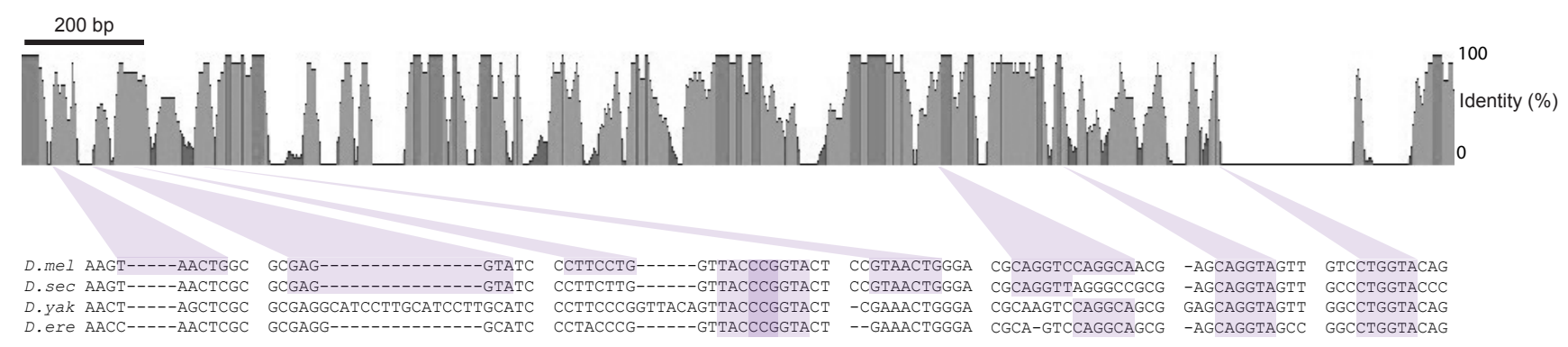

Figure S2. Multiple species alignment of the eveS2 minimal enhancer sequence with Zelda motifs and conservation noted. Highlighted Zelda motifs denote the minimal binding site sequence 5'-CAGGTA [40], allowing for one mismatch to identify possible low affinity Zelda binding sites $[56,18]$. 
bioRxiv preprint doi: https://doi.org/10.1101/101311; this version posted January 18,2017 . The copyright holder for this preprint (which was not certified by peer review) is the author/funder, who has granted bioRxiv a license to display the preprint in perpetuity. It is made available under aCC-BY-NC 4.0 International license.

Functional regulatory evolution outside of the minimal even-skipped stripe 2 enhancer $-10 / 12$

\section{Supplemental Construct Sequences}

$>$ D. erecta eveS2

CGGCGCAATATAACCCAATAATTTGAACCAACTCGCGGAGCAGCGAGGGCATCCTACCCGGTTACCCGGTACT GCATAACAATGAAACGAAACTGGGACAGATCGGTGATGGTTTCTCGCTGTGTGTGCCGTGTTAATCCGTTTGC CATCAGCCAGATTATTAGTCAATTGCAGTCGCAGTCGCAGTTGCAGTTGCAGGGTTTCGCTTTCCTCGTCCTCG TTTCACTTTCGAGTTAGACTTTATTGCAGCATCTTGCAGCAACAATCGGCGCAGTTTGGTAACACGCTGTGCCC TTCCACTTTCCACATTCCACGGCCCAATTCGGCGGATTTAGACGGAATCGAGGGACCCTGACTATGTTCGCATA ATGAAAGCGAAACCAAACCGGGTTGCAAAGTCAGGGCATTCCATCGCCGTCGCCATCGCCACCGCCATCTTCT GCGGGCGTTTGTTTGTTTGTTTGCTGGGATTAGCCAAGGGCTTGACTTGGAACCCAATCCCAATCCCAATCCCA ATCCCAAAGCCAATCCCAGTGCCCATCCCGATCGCAGTCCCATGCCCTTTTCATTAGAAAGTCATAAAAACAC ATAATAATGATGTCGAACGGATTAGCTGCGCGCAGTCCAGGCAGCGCAATTAACGGACTAGCAAACTGGGTT ATTTCTATTTTTTTATTTTCGCCGACTTAGCCCTGATCCGCGAGCTTAACCCGTTTGAGCCGCAGCAGGTAGCC ATCCCCATCCTGGCTTGCGCAAGTGGCAGGTTCGCTGCCCCAGGAGAGCCGTGGAGGCACTCTGGCCACTGGC CTGGTACAGTTGCCGCTGGGCATGATTATATCATCATAATAAATGTTT

$>$ D. erecta eveS2 large

TCCTCGAGGATTTCGGGCTGAATCACTTACTCAACCCGTCGATTCCGTCCGCGCAATCATCATAAATTCTCGGT CTTTTTGCTGTAATTGTTTTATGGCAGAAATTACTCAATCATCAAGCATAATTCCCTCGTTTTCGCCGTTTTATT GCCAATTTTTGCACTGCCTCCCGCCTTTGCCACTCCCGGCCCTTCCCTCATCGTTTTGCGAATCTCCGACGGATT CGCATTTCTATTGCGCGGACAATCCGGCCAGTGTGTTTGCCATTTACTTGCCATGATGACGGGCATAATCAGC GAGATCGGCGCTTTGTGAGTGCAGAATGTGCAATAAAGCGGCAACAATCGGCTTGGGATTCGCCTTCCCCTAT TCCCAGTATTGCCCGAGTGCCCGGACGACTGCGAAAGTGTTTGCGGATCGGGATCGGAATCGGAATAGGAAT AGGAATACGAGACTGAGCAGAGGCAGGTACTTCCCGCCGGCCGGACACTTTCGCCTAACCAAGCGGACTCAA CCCAACCCAACCCAACACAACCCAATCCAACCCACCCGATCGCCATAAAGGGTATTTACTGTCGCTGCCGCAG AGCCTCGCTTGACGACTTAACCCAAGCGGTCGTTCCACGTCCATTCTCCGGACGGAGTCAAAGACAAAGGCCG GCGGAGCTGGACAATAGGCAAGGTTGTTGCTTGTGGGTAGAGGGTTTCAATCCCGAAATTGTACCTTTCCCGG GCGAGAAGGGCTTGCATGTGGGCCTTTTCCAGGTCGCAGATGTAGGTAGATGTAGATGTAGATGCGGATGGG CCGGTCGAGTTAATGCCAATGCAAATTGCGGCGCAATATAACCCAATAATTTGAACCAACTCGCGGAGCAGC GAGGGCATCCTACCCGGTTACCCGGTACTGCATAACAATGAAACGAAACTGGGACAGATCGGTGATGGTTTCT CGCTGTGTGTGCCGTGTTAATCCGTTTGCCATCAGCCAGATTATTAGTCAATTGCAGTCGCAGTCGCAGTTGCA GTTGCAGGGTTTCGCTTTCCTCGTCCTCGTTTCACTTTCGAGTTAGACTTTATTGCAGCATCTTGCAGCAACAAT CGGCGCAGTTTGGTAACACGCTGTGCCCTTCCACTTTCCACATTCCACGGCCCAATTCGGCGGATTTAGACGG AATCGAGGGACCCTGACTATGTTCGCATAATGAAAGCGAAACCAAACCGGGTTGCAAAGTCAGGGCATTCCA TCGCCGTCGCCATCGCCACCGCCATCTTCTGCGGGCGTTTGTTTGTTTGTTTGCTGGGATTAGCCAAGGGCTTG ACTTGGAACCCAATCCCAATCCCAATCCCAATCCCAAAGCCAATCCCAGTGCCCATCCCGATCGCAGTCCCAT GCCCTTTTCATTAGAAAGTCATAAAAACACATAATAATGATGTCGAACGGATTAGCTGCGCGCAGTCCAGGCA GCGCAATTAACGGACTAGCAAACTGGGTTATTTCTATTTTTTTATTTTCGCCGACTTAGCCCTGATCCGCGAGC TTAACCCGTTTGAGCCGCAGCAGGTAGCCATCCCCATCCTGGCTTGCGCAAGTGGCAGGTTCGCTGCCCCAGG AGAGCCGTGGAGGCACTCTGGCCACTGGCCTGGTACAGTTGCCGCTGGGCATGATTATATCATCATAATAAAT GTTT

$>$ D. erecta eveS2+inter $3 / 7$

TTCGTTCCCAACTACGGCTAAGATATGCCAGTTTGTTTTGTCTCCGGCAATTATTGGAAATTTCATTGGGTCGA TTGGGTAGATGTCGATTGGGTCGATGTCGATGCCTTCCCTCGGGAAAAGTGAATAGGTTGTGCCATAAAAATC GCTGCTCTTGGAGATGAAATGCTGTAGTAGTATGCCAAAGCATCATTCTGCTTTTTTATTTTCTCACTGCTAAA TGCAGCTAATTTGTCGATTGTCTGAAAAGTGTTCTCTAAGCCGAAGCACTTTTTTATGATGTTGCTAGAAAATG AAATCACTTATGTTGCCATACATCCCCAGGCATTTTATGGCCATTTGAGTGCGGGGTGCGCAGTTCTGCTTAAG TGGCGGATGGAAACCACCACATTTATTCGAGGGATGATGTGCTCTAATACCTCCTCATCAAATGGGATGGTCT CTTCGCATGGAGAGTGGCAAACTCTTGGAAAAGTGAGGCGGAGTTAAAAAGCCGTGCTATATAACAAGATTT TTGATATTCAGTTATGTATATATGGACAAGAAATATCAAAGACCTTATCAAATATGTTGCCTTTTATCCTCGAA ATGAAACAAATGCTCTACTAATTTGGCAAGTCAAAAGATCAGTTCAGCAATTATTCAAAAGAACATAAAATAT GCGTATATTTTTGGGAATGTACCAGTGCTTTCCAAAATAGATTGCCAAACAAATCAACTAATAACTTTAATTTA AAAAACTGGGCAATCCTGAGTTGGCAGTCTTCCCAAGAATGGCTCCTCGAGGATTTCGGGCTGAATCACTTAC TCAACCCGTCGATTCCGTCCGCGCAATCATCATAAATTCTCGGTCTTTTTGCTGTAATTGTTTTATGGCAGAAA TTACTCAATCATCAAGCATAATTCCCTCGTTTTCGCCGTTTTATTGCCAATTTTTGCACTGCCTCCCGCCTTTGC CACTCCCGGCCCTTCCCTCATCGTTTTGCGAATCTCCGACGGATTCGCATTTCTATTGCGCGGACAATCCGGCC AGTGTGTTTGCCATTTACTTGCCATGATGACGGGCATAATCAGCGAGATCGGCGCTTTGTGAGTGCAGAATGT 
bioRxiv preprint doi: https://doi.org/10.1101/101311; this version posted January $18,2017$. The copyright holder for this preprint (which was not certified by peer review) is the author/funder, who has granted bioRxiv a license to display the preprint in perpetuity. It is made available under aCC-BY-NC 4.0 International license.

Functional regulatory evolution outside of the minimal even-skipped stripe 2 enhancer $-11 / 12$

GCAATAAAGCGGCAACAATCGGCTTGGGATTCGCCTTCCCCTATTCCCAGTATTGCCCGAGTGCCCGGACGAC TGCGAAAGTGTTTGCGGATCGGGATCGGAATCGGAATAGGAATAGGAATACGAGACTGAGCAGAGGCAGGTA CTTCCCGCCGGCCGGACACTTTCGCCTAACCAAGCGGACTCAACCCAACCCAACCCAACACAACCCAATCCAA CCCACCCGATCGCCATAAAGGGTATTTACTGTCGCTGCCGCAGAGCCTCGCTTGACGACTTAACCCAAGCGGT CGTTCCACGTCCATTCTCCGGACGGAGTCAAAGACAAAGGCCGGCGGAGCTGGACAATAGGCAAGGTTGTTG CTTGTGGGTAGAGGGTTTCAATCCCGAAATTGTACCTTTCCCGGGCGAGAAGGGCTTGCATGTGGGCCTTTTC CAGGTCGCAGATGTAGGTAGATGTAGATGTAGATGCGGATGGGCCGGTCGAGTTAATGCCAATGCAAATTGC GGCGCAATATAACCCAATAATTTGAACCAACTCGCGGAGCAGCGAGGGCATCCTACCCGGTTACCCGGTACT GCATAACAATGAAACGAAACTGGGACAGATCGGTGATGGTTTCTCGCTGTGTGTGCCGTGTTAATCCGTTTGC CATCAGCCAGATTATTAGTCAATTGCAGTCGCAGTCGCAGTTGCAGTTGCAGGGTTTCGCTTTCCTCGTCCTCG TTTCACTTTCGAGTTAGACTTTATTGCAGCATCTTGCAGCAACAATCGGCGCAGTTTGGTAACACGCTGTGCCC TTCCACTTTCCACATTCCACGGCCCAATTCGGCGGATTTAGACGGAATCGAGGGACCCTGACTATGTTCGCATA ATGAAAGCGAAACCAAACCGGGTTGCAAAGTCAGGGCATTCCATCGCCGTCGCCATCGCCACCGCCATCTTCT GCGGGCGTTTGTTTGTTTGTTTGCTGGGATTAGCCAAGGGCTTGACTTGGAACCCAATCCCAATCCCAATCCCA ATCCCAAAGCCAATCCCAGTGCCCATCCCGATCGCAGTCCCATGCCCTTTTCATTAGAAAGTCATAAAAACAC ATAATAATGATGTCGAACGGATTAGCTGCGCGCAGTCCAGGCAGCGCAATTAACGGACTAGCAAACTGGGTT ATTTCTATTTTTTTATTTTCGCCGACTTAGCCCTGATCCGCGAGCTTAACCCGTTTGAGCCGCAGCAGGTAGCC ATCCCCATCCTGGCTTGCGCAAGTGGCAGGTTCGCTGCCCCAGGAGAGCCGTGGAGGCACTCTGGCCACTGGC CTGGTACAGTTGCCGCTGGGCATGATTATATCATCATAATAAATG

$>$ D. erecta eveS $2+\mathrm{S} 3 / 7$

GGACACAAGGATCCTCGAAATCGAGAGCGACCTCGCTGCATTAGAAAACTAGATCAGTTTTTTGTTTCGCGTC CGCTGATTTTTGTGCCCGTTTGCTCTCTTTACGGTTTATGGCCCCGTTTCCATTATTTCGTCATTTTTCCACATTT CCCAGCTCCTTTGTGCCGCTCAAAGAAATCTGTACGGAATTATGGTATATGCAGATTTTTATGGGTCGCCGATC CGGTTCGCGGAACGCGAGTGTCCTGCCGCGAGGACCTCAGCGGCGATCCTTGTCGGCCGTATTAAGAAAGTA GATCACGTTTTTTGTTCCCATTGTGCGCTTTTTTCGCTGCGCCAGTTCTGTTCCCCGAACCCAGCGAACTGCTCT AATTTTTTAATTCTTCACGGACTTTCATTGGGCTCCTGGAAAAACACGCACAAGGCTAGCTCTAGGACTCTACT GGCATACCTGTAATGGTGTCCATAACGCGCACTGCTCTCGTTTTTAAGACCCGTTTGTGTTCGTCTCTGGAAAG TAGATGGCATTCACATTTTTATGAGTTCGTTCCCAACTACGGCTAAGATATGCCAGTTTGTTTTGTCTCCGGCA ATTATTGGAAATTTCATTGGGTCGATTGGGTAGATGTCGATTGGGTCGATGTCGATGCCTTCCCTCGGGAAAA GTGAATAGGTTGTGCCATAAAAATCGCTGCTCTTGGAGATGAAATGCTGTAGTAGTATGCCAAAGCATCATTC TGCTTTTTTATTTTCTCACTGCTAAATGCAGCTAATTTGTCGATTGTCTGAAAAGTGTTCTCTAAGCCGAAGCA CTTTTTTATGATGTTGCTAGAAAATGAAATCACTTATGTTGCCATACATCCCCAGGCATTTTATGGCCATTTGA GTGCGGGGTGCGCAGTTCTGCTTAAGTGGCGGATGGAAACCACCACATTTATTCGAGGGATGATGTGCTCTAA TACCTCCTCATCAAATGGGATGGTCTCTTCGCATGGAGAGTGGCAAACTCTTGGAAAAGTGAGGCGGAGTTAA AAAGCCGTGCTATATAACAAGATTTTTGATATTCAGTTATGTATATATGGACAAGAAATATCAAAGACCTTAT CAAATATGTTGCCTTTTATCCTCGAAATGAAACAAATGCTCTACTAATTTGGCAAGTCAAAAGATCAGTTCAG CAATTATTCAAAAGAACATAAAATATGCGTATATTTTTGGGAATGTACCAGTGCTTTCCAAAATAGATTGCCA AACAAATCAACTAATAACTTTAATTTAAAAAACTGGGCAATCCTGAGTTGGCAGTCTTCCCAAGAATGGCTCC TCGAGGATTTCGGGCTGAATCACTTACTCAACCCGTCGATTCCGTCCGCGCAATCATCATAAATTCTCGGTCTT TTTGCTGTAATTGTTTTATGGCAGAAATTACTCAATCATCAAGCATAATTCCCTCGTTTTCGCCGTTTTATTGCC AATTTTTGCACTGCCTCCCGCCTTTGCCACTCCCGGCCCTTCCCTCATCGTTTTGCGAATCTCCGACGGATTCGC ATTTCTATTGCGCGGACAATCCGGCCAGTGTGTTTGCCATTTACTTGCCATGATGACGGGCATAATCAGCGAG ATCGGCGCTTTGTGAGTGCAGAATGTGCAATAAAGCGGCAACAATCGGCTTGGGATTCGCCTTCCCCTATTCC CAGTATTGCCCGAGTGCCCGGACGACTGCGAAAGTGTTTGCGGATCGGGATCGGAATCGGAATAGGAATAGG AATACGAGACTGAGCAGAGGCAGGTACTTCCCGCCGGCCGGACACTTTCGCCTAACCAAGCGGACTCAACCC AACCCAACCCAACACAACCCAATCCAACCCACCCGATCGCCATAAAGGGTATTTACTGTCGCTGCCGCAGAGC CTCGCTTGACGACTTAACCCAAGCGGTCGTTCCACGTCCATTCTCCGGACGGAGTCAAAGACAAAGGCCGGCG GAGCTGGACAATAGGCAAGGTTGTTGCTTGTGGGTAGAGGGTTTCAATCCCGAAATTGTACCTTTCCCGGGCG AGAAGGGCTTGCATGTGGGCCTTTTCCAGGTCGCAGATGTAGGTAGATGTAGATGTAGATGCGGATGGGCCG GTCGAGTTAATGCCAATGCAAATTGCGGCGCAATATAACCCAATAATTTGAACCAACTCGCGGAGCAGCGAG GGCATCCTACCCGGTTACCCGGTACTGCATAACAATGAAACGAAACTGGGACAGATCGGTGATGGTTTCTCGC TGTGTGTGCCGTGTTAATCCGTTTGCCATCAGCCAGATTATTAGTCAATTGCAGTCGCAGTCGCAGTTGCAGTT GCAGGGTTTCGCTTTCCTCGTCCTCGTTTCACTTTCGAGTTAGACTTTATTGCAGCATCTTGCAGCAACAATCG GCGCAGTTTGGTAACACGCTGTGCCCTTCCACTTTCCACATTCCACGGCCCAATTCGGCGGATTTAGACGGAAT CGAGGGACCCTGACTATGTTCGCATAATGAAAGCGAAACCAAACCGGGTTGCAAAGTCAGGGCATTCCATCG 
bioRxiv preprint doi: https://doi.org/10.1101/101311; this version posted January 18,2017 . The copyright holder for this preprint (which was not certified by peer review) is the author/funder, who has granted bioRxiv a license to display the preprint in perpetuity. It is made available under aCC-BY-NC 4.0 International license.

Functional regulatory evolution outside of the minimal even-skipped stripe 2 enhancer $-12 / 12$

CCGTCGCCATCGCCACCGCCATCTTCTGCGGGCGTTTGTTTGTTTGTTTGCTGGGATTAGCCAAGGGCTTGACT TGGAACCCAATCCCAATCCCAATCCCAATCCCAAAGCCAATCCCAGTGCCCATCCCGATCGCAGTCCCATGCC CTTTTCATTAGAAAGTCATAAAAACACATAATAATGATGTCGAACGGATTAGCTGCGCGCAGTCCAGGCAGCG CAATTAACGGACTAGCAAACTGGGTTATTTCTATTTTTTTATTTTCGCCGACTTAGCCCTGATCCGCGAGCTTA ACCCGTTTGAGCCGCAGCAGGTAGCCATCCCCATCCTGGCTTGCGCAAGTGGCAGGTTCGCTGCCCCAGGAGA GCCGTGGAGGCACTCTGGCCACTGGCCTGGTACAGTTGCCGCTGGGCATGATTATATCATCATAATAAATGTT $\mathrm{T}$

$>$ D. erecta eveS2 large del. Zelda

TCCTCGAGGATTTCGGGCTGAATCACTTACTCAACCCGTCGATTCCGTCCGCGCAATCATCATAAATTCTCGGT CTTTTTGCTGTAATTGTTTTATGGCAGAAATTACTCAATCATCAAGCATAATTCCCTCGTTTTCGCCGTTTTATT GCCAATTTTTGCACTGCCTCCCGCCTTTGCCACTCCCGGCCCTTCCCTCATCGTTTTGCGAATCTCCGACGGATT CGCATTTCTATTGCGCGGACAATCCGGCCAGTGTGTTTGCCATTTACTTGCCATGATGACGGGCATAATCAGC GAGATCGGCGCTTTGTGAGTGCAGAATGTGCAATAAAGCGGCAACAATCGGCTTGGGATTCGCCTTCCCCTAT TCCCAGTATTGCCCGAGTGCCCGGACGACTGCGAAAGTGTTTGCGGATCGGGATCGGAATCGGAATAGGAAT AGGAATACGAGACTGAGCAGAGGAATTTACTTCCCGCCGGCCGGACACTTTCGCCTAACCAAGCGGACTCAA CCCAACCCAACCCAACACAACCCAATCCAACCCACCCGATCGCCATAAAGGGTATTTACTGTCGCTGCCGCAG AGCCTCGCTTGACGACTTAACCCAAGCGGTCGTTCCACGTCCATTCTCCGGACGGAGTCAAAGACAAAGGCCG GCGGAGCTGGACAATAGGCAAGGTTGTTGCTTGTGGGTAGAGGGTTTCAATCCCGAAATTGTACCTTTCCCGG GCGAGAAGGGCTTGCATGTGGGCCTTTTCAATTTCGCAGATGAATTTAGATGTAGATGTAGATGCGGATGGGC CGGTCGAGTTAATGCCAATGCAAATTGCGGCGCAATATAACCCAATAATTTGAACCAACTCGCGGAGCAGCG AGGGCATCCTACCCGGTTACCCGGTACTGCATAACAATGAAACGAAACTGGGACAGATCGGTGATGGTTTCTC GCTGTGTGTGCCGTGTTAATCCGTTTGCCATCAGCCAGATTATTAGTCAATTGCAGTCGCAGTCGCAGTTGCAG TTGCAGGGTTTCGCTTTCCTCGTCCTCGTTTCACTTTCGAGTTAGACTTTATTGCAGCATCTTGCAGCAACAATC GGCGCAGTTTGGTAACACGCTGTGCCCTTCCACTTTCCACATTCCACGGCCCAATTCGGCGGATTTAGACGGA ATCGAGGGACCCTGACTATGTTCGCATAATGAAAGCGAAACCAAACCGGGTTGCAAAGTCAGGGCATTCCAT CGCCGTCGCCATCGCCACCGCCATCTTCTGCGGGCGTTTGTTTGTTTGTTTGCTGGGATTAGCCAAGGGCTTGA CTTGGAACCCAATCCCAATCCCAATCCCAATCCCAAAGCCAATCCCAGTGCCCATCCCGATCGCAGTCCCATG CCCTTTTCATTAGAAAGTCATAAAAACACATAATAATGATGTCGAACGGATTAGCTGCGCGCAGTCCAGGCAG CGCAATTAACGGACTAGCAAACTGGGTTATTTCTATTTTTTTATTTTCGCCGACTTAGCCCTGATCCGCGAGCT TAACCCGTTTGAGCCGCAGCAGGTAGCCATCCCCATCCTGGCTTGCGCAAGTGGCAGGTTCGCTGCCCCAGGA GAGCCGTGGAGGCACTCTGGCCACTGGCCTGGTACAGTTGCCGCTGGGCATGATTATATCATCATAATAAATG TTT

$>$ D. erecta eveS2 $+3 X$ Zelda

GAGGCAGGTACTTCCTTTCCAGGTCGCAGATGTAGGTAGATGTCGGCGCAATATAACCCAATAATTTGAACCA ACTCGCGGAGCAGCGAGGGCATCCTACCCGGTTACCCGGTACTGCATAACAATGAAACGAAACTGGGACAGA TCGGTGATGGTTTCTCGCTGTGTGTGCCGTGTTAATCCGTTTGCCATCAGCCAGATTATTAGTCAATTGCAGTC GCAGTCGCAGTTGCAGTTGCAGGGTTTCGCTTTCCTCGTCCTCGTTTCACTTTCGAGTTAGACTTTATTGCAGC ATCTTGCAGCAACAATCGGCGCAGTTTGGTAACACGCTGTGCCCTTCCACTTTCCACATTCCACGGCCCAATTC GGCGGATTTAGACGGAATCGAGGGACCCTGACTATGTTCGCATAATGAAAGCGAAACCAAACCGGGTTGCAA AGTCAGGGCATTCCATCGCCGTCGCCATCGCCACCGCCATCTTCTGCGGGCGTTTGTTTGTTTGTTTGCTGGGA TTAGCCAAGGGCTTGACTTGGAACCCAATCCCAATCCCAATCCCAATCCCAAAGCCAATCCCAGTGCCCATCC CGATCGCAGTCCCATGCCCTTTTCATTAGAAAGTCATAAAAACACATAATAATGATGTCGAACGGATTAGCTG CGCGCAGTCCAGGCAGCGCAATTAACGGACTAGCAAACTGGGTTATTTCTATTTTTTTATTTTCGCCGACTTAG CCCTGATCCGCGAGCTTAACCCGTTTGAGCCGCAGCAGGTAGCCATCCCCATCCTGGCTTGCGCAAGTGGCAG GTTCGCTGCCCCAGGAGAGCCGTGGAGGCACTCTGGCCACTGGCCTGGTACAGTTGCCGCTGGGCATGATTAT ATCATCATAATAAATGTTT 